\title{
Overpressure Distribution of Special Stiffened Plates
}

\author{
ZHANG Mi ${ }^{1, a}$, WU Guo Min ${ }^{2, b}$ \\ ${ }^{1}$ China Ship Development and Design Center, Wuhan, China \\ ${ }^{2}$ China Ship Development and Design Center, Wuhan, China \\ a zhangmi2129@foxmail.com, ${ }^{b}$ wugm20@163.com
}

Keywords: blast wave, overpressure, stiffened plates, LS-DYNA

\begin{abstract}
This article shows ten special stiffened plates. With the help of high transient non-linear numerical software LS-DYNA, air blast wave load can impulse on stiffened plates. Overpressure of air blast wave distributes on the stiffened plates with different kinds of forms. The overpressure distributions are effected by the stiffeners. From the results, it can be seen that stiffeners have the effects of reflection and convergence. The stiffening ways of facing to the explosive can reduce the quasi-static pressure with small mass of TNT and small size of plates. Stiffeners can be used in some engineering structures to protect important parts.
\end{abstract}

\section{Introduction}

The structures of warships are inclined to be attacked with air-blast waves. The structures are almost made up of stiffened plates, so the research on the warships' strength is almost actually on the research of the stiffened plates which sometimes are under blast wave's attack. The load of air blast wave is one type of dynamic loadings which can lead to the plastic zones on the stiffened plates. The methods on the research of air blast loading have changed from experimental ways to the combined ways which use experiments and numerical methods with the development of numerical software. The high transient non-linear numerical software is accepted widely for their good results in calculations and lower cost compared with experiments.

This article is focused on some special types of stiffened plates to study the influence of stiffeners of the plates on the distribution of air blast wave. The numerical method is used in this research to detect the over-pressure of selected points to analysis the function of stiffeners. The analytical results may give some advice to engineering practice of how to design the stiffened plates.

\section{Plate Geometry and Numerical Modelling}

Plate Geometry. The stiffened plates used are $40 \mathrm{~cm} * 30 \mathrm{~cm}$ in size with uniform thickness of $0.35 \mathrm{~cm}$ as shown in Figure 1. The stiffeners are rectangular in cross section with $5 \mathrm{~cm}$ in height and with uniform thickness of $0.54 \mathrm{~cm}$. The stiffener size is commonly used to stiffen the plates in ship hull structures [1,2]. In the present study, an unstiffened plate are stiffened with ten different stiffeners configurations ( $\mathrm{S} 1$ to $\mathrm{S} 10$ ) as shown in Figure 2.

Numerical Modeling. As some stiffeners would endure the blast wave, shell elements are chosen to format plates and stiffeners in numerical model. In addition, as one single plate is chosen to simulate the real ship hull structure, clamped boundary condition are also chosen to ensure the accuracy of the simulation results. ALE method considering the coupling of air and structure is selected to simulate the process of air blast load acting on plates. Therefore, the zone of air with the size of $50 \mathrm{~cm} * 40 \mathrm{~cm} * 55 \mathrm{~cm}$ is modeled in LS-DYNA. The model of stiffened plate is inside of the air zone and the nodes of their finite model is merged together to deliver mechanical parameters. 


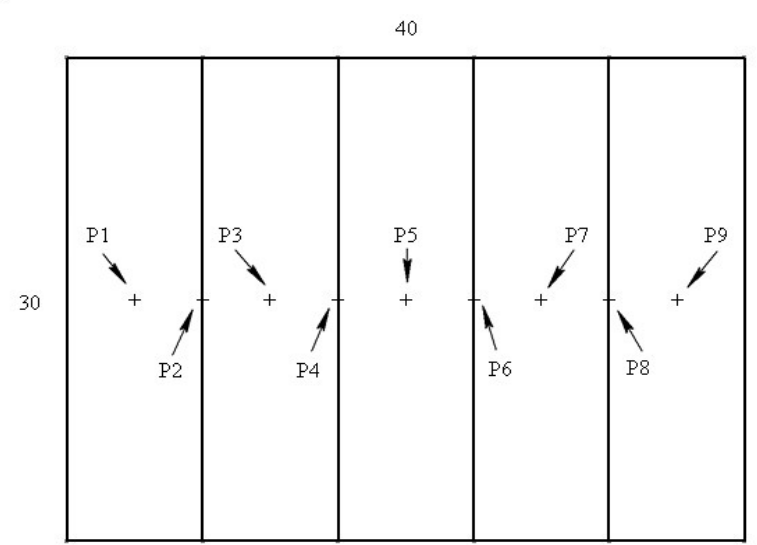

Fig.1 The size of stiffened plates
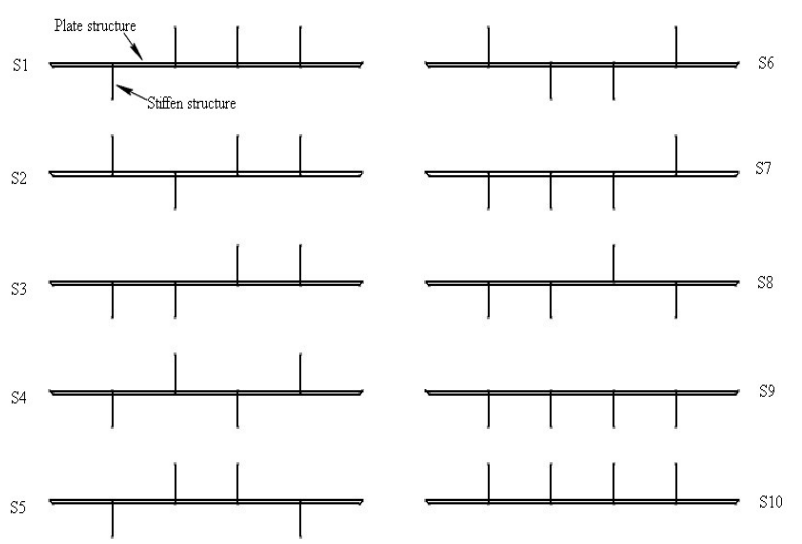

Fig.2 Ten different stiffeners configurations

Model Parameters. The center of detonation is in the center of the plate with the height of 20 centimeters. The air blast load is produced by TNT with the weight of 2.24 kilograms. The density of TNT is 1,630 kilograms per stere and detonation velocity of TNT is 0.67174 centimeters per microseconds. The CJ pressure of TNT is 0.18503 Mbar.

The equation state of TNT is chosen as Equation 1,

$$
p=A_{1}\left(1-\frac{\omega}{R_{1} V}\right) e^{-R_{1} V}+B_{1}\left(1-\frac{\omega}{R_{2} V}\right) e^{-R_{2} V}+\frac{\omega E}{V}
$$

where $\mathrm{p}$ is the pressure of air blast wave, $\mathrm{V}$ is the relevant volume, and $\mathrm{A}_{1}, \mathrm{~B}_{1}, \mathrm{R}_{1}, \mathrm{R}_{2}, \omega$ are property numbers of TNT.

The parameters of plates are shown in Table 1 , where $\rho$ is the density of plate, $G$ is the shear elasticity, $A$ is constant, $E_{0}$ is the initial inner energy and $V_{0}$ is the initial relative volume. Johnson-Cook material model and equation state of Gruneisen are selected as basic properties of plates when simulating. Stiffeners of the plates share the same parameters of plates which have not much effect on the results which this article cares about.

Table 1 The material parameters of stiffened plate structure

\begin{tabular}{cccccc}
\hline Parameter & $\rho /\left[\mathrm{g} / \mathrm{cm}^{3}\right]$ & $\mathrm{G} /[\mathrm{GPa}]$ & $\mathrm{A}$ & $\mathrm{E}_{0}$ & $\mathrm{~V}_{0}$ \\
\hline Value & 7.83 & 0.77 & 0.43 & 0 & 1.0 \\
\hline
\end{tabular}

\section{Results and Discussions}

Numerical analysis carried out in the present investigation aims to study the dissemination of blast in air and its value in some special place of stiffened plate. Nine special observation points (P1-P9) are set that can be seen in Figure 1. It aims to study overpressure of coupling face between air and plates. The overpressure of coupling face is influenced by different kinds of stiffened ways. When TNT explodes above the subject, blast wave will propagate in all directions, and at some point, the wave will reach the subject and hit it. With time going on, the blast wave will reflect on the ground formed face of reflection. The wave of reflection has an angle with ground, and if the angle is smaller than a value, it's called normal reflection. Otherwise, it's called the Mach wave [3]. The incoming and reflected waves will overlay with each other and forced the subject together. Considering the stiffeners on plates, it can be easily seen that stiffeners will disturb the distribution of air blast wave.

There are two kinds of normal configurations-S9 and S10 which used widely in engineering [4].Therefore, overpressure in P1 to P9 of S9 and S10 can set as references when study other results. According to configuration of stiffened plates, results of S1, S2 and S3 are combined together to compare with S9 and S10, as the figure can be seen in Figure 3. 


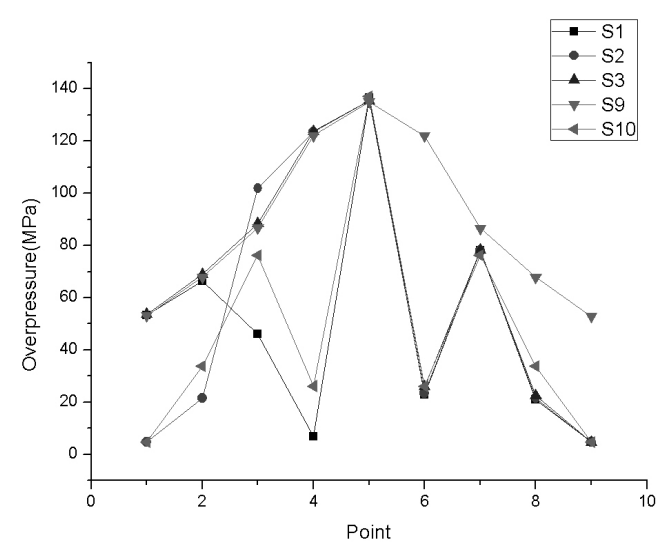

Fig. 3 Overpressure results in condition of S1, S2, S3, S9 and S10

Plates of S1, S2 and S3 have the same stiffened ways in right side of configurations. From Figure 3, it can be seen that condition S1 is different from condition S10 on the left side of configuration which infers the reflection and convergence of blast wave are inclined in the left side of the plate with no stiffeners. Thus, overpressure on P8 of condition S1 is less than the same place of condition S10. In condition $\mathrm{S} 1$, incoming waves make the pressure of $\mathrm{P} 2$ increase and overlay with reflected waves, and quasi-static pressure is formed in P8 of condition S1. However, P7 of condition S1 formed no quasi-static pressure for no stiffeners and repeatedly reflecting. Location of P8 is worth of attention for it away from the detonation point. In addition, with the small angle of reflected wave with ground, Mach wave are not formed in P8 and all reflection waves are normal waves. From Figure 3, it can be also seen that value of P5 to P10 are the same for their same stiffened ways in the right side of configuration.

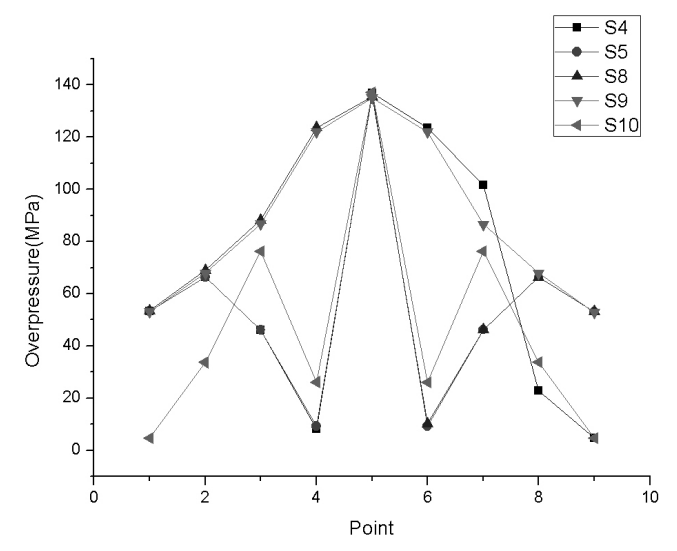

Fig. 4 Overpressure results in condition of S4, S5, S8, S9 and S10

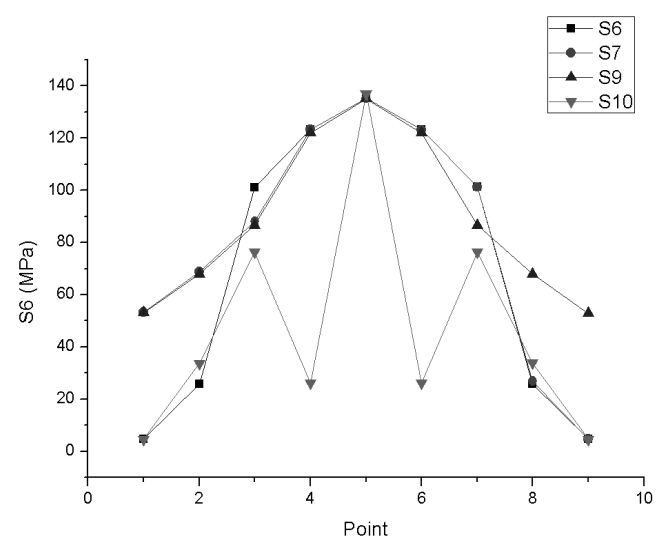

Fig. 5 Overpressure results in condition of S6, S7, S9 and S10 
Plates of S4, S5 and S8 have the same stiffened ways in some part of configurations. From Figure 4 , it can be seen that the value of overpressure of condition S4 are the same as condition S5 on the left side of the plates. The values of overpressure of condition $S 9$ are distributed well on the stiffened plate, which reduce according to the distance to center point of detonation. However, stiffeners are to the benefit of blast wave reflection, which results in overpressure in P6 of condition S5 and S10 is less than condition S9. It can be seen that overpressure of P8 in condition S10 is much more than the pressure in P6 and P7, which can be inferred that stiffeners are good for blast wave reflection.

From Figure 5, it can be seen that the value in condition S6 of P3 and P7 is higher than condition S9, and the value in condition S7 of P8 is less than condition S10. Considering the distance away from the center of detonation, the stiffeners are good for the reflection and convergence of blast wave, which makes the value of points closed to the center of detonation higher than the points closed to the margin.

\section{Conclusions}

Numerical analysis has been carried out to investigate the effects of stiffener configuration. The study mainly cared about the stiffeners effects on how to reflect and converge the blast wave. The following conclusions are drawn from the present study:

The stiffening ways of facing to the explosive (condition S10) shows some differences comparing with the normal ways of stiffening (condition S9). The value of condition S10 in less than condition S9, which refers those stiffeners are good for the reflection of blast waves. According to needs of reality, proper stiffeners can be added to the plate to reduce the effects of overpressure.

From the results, it can be seen that the P5 in all conditions have almost the same value. The distance between this point and the center of detonation is the smallest. It infers that overpressure of the location closing to the center of detonation is not effected by the configuration of plate.

From the overpressure results, it can be seen that the value of condition S10 is the smallest than compared with other conditions. Therefore, it refers that with small mass of TNT and small size of plate, the stiffening ways of facing to explosive can be used to enhance strength of structures.

\section{Acknowledgements}

This research forms part of the master's research by the lead author at the China Ship Development and Design Center (CSDDC), Mi Zhang, China under the guidance of the tutor. The author wishes to thank the tutor, senior engineer of CSDDC and the assistant tutor, also the senior engineer of CSDDC. Last but not least, the author appreciates all the help from the CSDDC and schoolmates during this study.

\section{References}

[1] HOU Hai liang, ZHU Xi, GU Mei bang, in: Study on failure mode of stiffened plate and optimized design of structure subjected to blast load. Explosive and Shock Waves. Vol. 27 (2007), p.26.

[2] Slater J E, Houlston R, Ritizel D V. Air blast studies on naval steel panels, final report [R]. Task DMEM-53, Defence Research Establishment Suffield Report No. 505, Ralston, Albert, Canada, 1990.

[3] Henrych J: The dynamics of explosion and its use. Translated by Xiong Jianguo. (Beijing: Science Press, 1987).

[4] MEI Zhi-yuan, in: Dynamic response researches of ship's stiffened plate structure under explosive load. Explosive and Shock Waves. Vol. 24 (2004), p.82. 